\title{
Robust Damping of Multiple Swing Modes Employing Global Stabilizing Signals With a TCSC
}

\author{
Balarko Chaudhuri, Student Member, IEEE, and Bikash C. Pal, Senior Member, IEEE
}

\begin{abstract}
This paper demonstrates a robust damping control design for multiple swing mode damping in a typical power system model using global stabilizing signals. A multiple-input, single-output (MISO) controller is designed for a thyristor-controlled series capacitor (TCSC) to improve the damping of the critical interarea modes. The stabilizing signals are obtained from remote locations based on observability of the critical modes. A $\mathcal{H}_{\infty}$ damping control design based on the mixed-sensitivity formulation in a linear matrix inequality (LMI) framework is carried out. It is shown that, with local signal, supplementary damping control through three flexible AC transmission systems (FACTS) devices is necessary to provide damping to the three dominant interarea modes. On the other hand, the use of global signals has been shown to improve the damping of all the critical interarea modes with a single controller for the TCSC only. The damping performance of the centralized controller was examined in the frequency and the time domain for various operating scenarios. The controller was found to be robust against varying power-flow patterns, load characteristics, tie-line strengths, and system nonlinearities, including saturation.
\end{abstract}

Index Terms-Centralized control, decentralized control, $\mathbf{H}$-infinity control, reduced order system, robustness.

\section{INTRODUCTION}

I NTERAREA oscillations $(0.2-1.0 \mathrm{~Hz})$ are inherent in large interconnected power systems [1]. System outage resulting from these oscillations is of growing concern [1]. Over the last three decades, attention has been focused on power system damping control design to reduce the risks of system outage following undesirable oscillations.

The traditional approach to damp out interarea oscillations is through installation of power system stabilizers (PSS) [2] that provide supplementary control action through the excitation system of generators. In recent times, the use of FACTS devices has become a common practice to make full utilization of existing transmission capacities instead of adding new lines which are often restricted for economic and environmental reasons [3]. In addition to enhancing the available transfer capacity (ATC) of the transmission system, supplementary control is being added to these FACTS devices to damp out the interarea oscillations.

One of the major concerns in a practical system is that the number of dominant interarea modes is often larger than the number of control devices available to control them [4]. In recent years, much research is, therefore, focussed on designing

Manuscript received May 2, 2003. This work is supported by in part by ABB, USA and in part by EPSRC, U.K. under Grant GR/R/31676.

The authors are with the Department of Electrical and Electronic Engineering at Imperial College, London SW7 2BT, U.K. (e-mail: b.chaudhuri@imperial.ac.uk; b.pal@imperial.ac.uk).

Digital Object Identifier 10.1109/TPWRS.2003.821463 new control structures which can improve the damping of multiple swing modes. The primary idea behind the control design is to employ a combination of remote stabilizing signals with diverse modal contents. The remote stabilizing signals are often referred to as "global signals" to illustrate that they contain information about overall network dynamics as opposed to local control signal which lacks adequate observability of some of the significant interarea modes [5]. For local modes, the largest residue is associated with a local signal (e.g., generator rotor speed signal for a PSS). But for interarea modes, the local signal may not be the one with maximum observability. The signal with maximum observability for a particular mode may be derived from a remote location or as a combination of signals from several locations. The recent advances in wide-area measurement (WAM) technologies using phasor measurement units (PMUs) can deliver synchronous phasors and control signals at a high speed (e.g., at a 30-Hz sampling rate) [5], [6]. It is possible to deploy PMUs at strategic locations on the grid and obtain a coherent picture of the entire network in real time [6]. The PMUs measure positive sequence voltage and currents at different locations of the grid and can deliver the measurements as frequently as once per cycle of power frequency. The latest state of the art of global positioning system (GPS) technology ensures proper time synchronization among several global signals [6]. Reference [7] has suggested a new information architecture to transmit real-time operating data and control signals. From an economic viewpoint, implementation of the centralized controllers using global signals may be more cost effective than installing new control devices [4]. Reference [8] has proposed a two-level control design for PSS and static VAr compensator (SVC) using global signals. Reference [4] has shown that an optimum and weighted combination of local and global signals could successfully be used for the control design of PSS and TCSC. The basic idea was to illustrate the benefit of combining input signals to move the system zeros to desirable locations.

In this work, we propose a multivariable control design methodology for robust damping of interarea oscillations employing the local as well as the remote stabilizing signals. Local signal in the form of real power flow in the line adjacent to a FACTS device has been used for decentralized design of the damping controllers for a TCSC, SVC, and a controllable phase shifter (CPS). A sequential loop closure approach has been adopted for this decentralized design. Real power flow signals from three remote locations were also used to design a centralized three-input, single-output controller for a TCSC. The role of a centralized controller using remote measurements to damp out multiple swing modes with a single device is explored in this paper. 


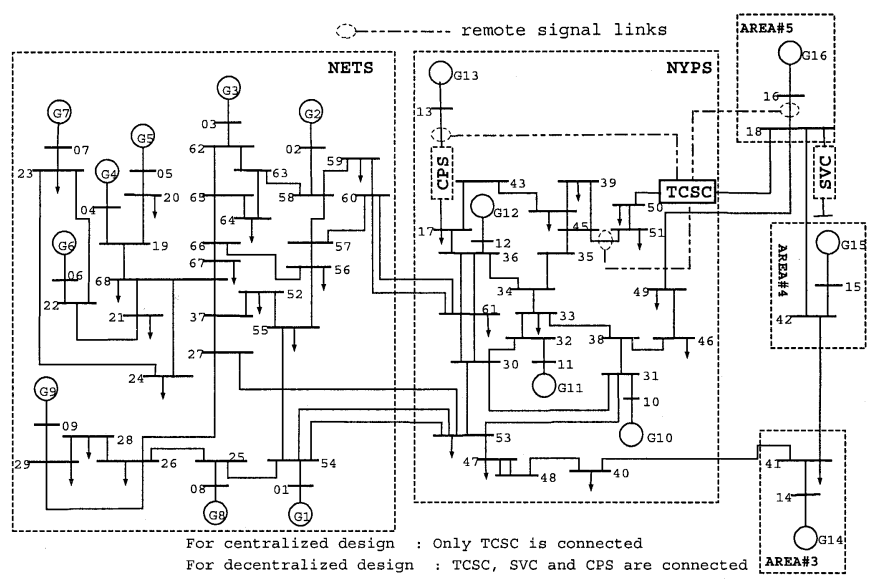

Fig. 1. Sixteen-machine five-area study system.

\section{STUDY SYSTEM}

A 16-machine, five-area study system, shown in Fig. 1, was considered for the damping control design. This is a reducedorder model of the New England and New York interconnected system. The first nine machines (G1 to G9) are the simple representation of the New England Test System (NETS) generation while machines G10 to G13 represent the New York Power System (NYPS). The last three machines G14 to G16 are the dynamic equivalents of the three neighboring areas connected to the NYPS. All of the machines were modeled with four windings on the rotor (i.e., field circuit), one damper in the d-axis and two dampers in the q-axis. The first eight machines have slow excitation (IEEE-type DC1A) while machine G9 is equipped with a fast-acting static excitation system (IEEE ST1A) [2] and a speed-input power system stabilizer to ensure adequate damping for its local modes. The rest of the machines were under manual excitation control. The loads were all assumed to be of constant impedance type for the base case. The detailed bus data, line data, and the dynamic characteristics for the machine, exciters, and loads can be found in [9] and [10]. NYPS is required to import $1550 \mathrm{MW}$ from Area \#5 and to facilitate this power transfer, a TCSC was installed in the line between bus \#18 and \#50. For this power-flow scenario, the compensation of the TCSC was calculated to be $50 \%$. A centralized design of the supplementary damping controller using remote stabilizing signals was carried out for the TCSC.

To demonstrate the decentralized design, two additional FACTS devices were considered at the following locations. To facilitate a large power transfer (3000 MW) through the line connecting buses \#13-\#17, a controllable phase shifter (CPS), with a steady-state setting of 10 degrees, was placed in this line. One SVC was also installed at bus \#18 to maintain an acceptable voltage profile throughout the system under this stressed power-flow condition. The output of the SVC was required to be 117 MVAR.

The prefault steady-state operation of the systems assumes a double circuit tie between bus \#53 and \#54 and the outage of one of these circuits takes the system into postfault steady state. The results of eigenanalysis, displayed in Table I, confirm the presence of four interarea modes out of which the first three
TABLE I

DAMPING Ratios and FReQuencies OF INTERAREA Modes With CENTRALIZED CONTROL FOR TCSC USING GLOBAL SIGNALS

\begin{tabular}{c|c|c|c|c|c|c|c|c}
\hline \multirow{2}{*}{ Mode No. } & \multicolumn{4}{|c|}{ Pre-fault } & \multicolumn{4}{c}{ Post-fault } \\
\cline { 2 - 9 } & \multicolumn{2}{|c|}{ Open-loop } & \multicolumn{2}{c|}{ Closed-loop } & \multicolumn{3}{c}{ Open-loop } & \multicolumn{2}{c}{ Closed-loop } \\
\cline { 2 - 9 } & $\zeta$ & $\mathrm{f}(\mathrm{Hz})$ & $\zeta$ & $\mathrm{f}(\mathrm{Hz})$ & $\zeta$ & $\mathrm{f}(\mathrm{Hz})$ & $\zeta$ & $\mathrm{f}(\mathrm{Hz})$ \\
\hline 1 & $\mathbf{0 . 0 6 2 6}$ & 0.3262 & $\mathbf{0 . 3 5 4 5}$ & 0.3401 & $\mathbf{0 . 0 5 0 7}$ & 0.3728 & $\mathbf{0 . 2 7 1 6}$ & 0.3433 \\
\hline 2 & $\mathbf{0 . 0 4 3 5}$ & 0.5080 & $\mathbf{0 . 1 3 3 0}$ & 0.4853 & $\mathbf{0 . 0 4 2 3}$ & 0.5071 & $\mathbf{0 . 1 2 5 8}$ & 0.5313 \\
\hline 3 & $\mathbf{0 . 0 5 5 4}$ & 0.6232 & $\mathbf{0 . 1 1 2 0}$ & 0.6116 & $\mathbf{0 . 0 4 6 8}$ & 0.5937 & $\mathbf{0 . 1 5 6 4}$ & 0.5485 \\
\hline 4 & 0.0499 & 0.7915 & 0.0518 & 0.7909 & 0.0499 & 0.7912 & 0.0520 & 0.7905 \\
\hline
\end{tabular}

are critical, necessitating damping control action. Mode \#4, on its own, settles in less than $10 \mathrm{~s}$ as its frequency $(0.79 \mathrm{~Hz})$ is comparatively higher than the other modes (the higher the oscillation frequency, the faster is the settling for a given damping ratio). Since the influence of this mode on interarea oscillation does not last beyond $10 \mathrm{~s}$ and an overall system settling time of 10-12 s is perfectly acceptable, it is not required to provide additional damping to this mode. Therefore, it was decided to provide supplementary damping control action to the first three critical interarea modes only. A modal observability analysis [11] was carried out to identify the most effective stabilizing signals for it. The results of the observability analysis revealed that $P_{51,45}, P_{18,16}$, and $P_{13,17}$ were the most effective signals where $P_{51,45}, P_{18,16}$, and $P_{13,17}$ indicate the power flow in the lines between buses \#51-\#45, buses \#18-\#16, and buses \#13-\#17, respectively. Modal analysis [11] considering all of the signals available throughout the system had shown that the interarea modes are mostly observable in the real power flow in these lines.

\section{MODEL OF TCSC}

The power injection model, suggested in [12] for different FACTS devices, was used for the TCSC. In this modeling approach, the effect of the FACTS devices on the power flow is represented as variable power injection at the terminal buses of the lines. The power injection varies with the FACTS control parameters. This process requires no modification of the bus admittance matrix during the power-flow iterations. Fig. 2 shows a typical power injection model of a TCSC connected in the line between bus $k$ and $m$. The series capacitor $\left(j X_{C}\right)$ is initially represented as a current $(\bar{I})$ dependent voltage $\left(\bar{V}_{\text {se }}=\right.$ $\left.-j X_{C} \bar{I}\right)$ source, which is later transformed into a current source $\left(\bar{I}_{s}=\left(\bar{V}_{s e} / j X_{\mathrm{km}}\right)\right)$ in parallel with the line. The effect of the current source is subsequently replaced by equivalent power injections $P_{k}, Q_{k}, P_{m}$, and $Q_{m}$ given by

$$
\begin{aligned}
P_{k} & =\frac{k_{c}}{\left(k_{c}-1\right)} V_{k} V_{m} B_{\mathrm{km}} \sin \left(\theta_{k}-\theta_{m}\right) \\
Q_{k} & =\frac{k_{c}}{\left(k_{c}-1\right)} B_{\mathrm{km}}\left[V_{k}^{2}-V_{k} V_{m} \cos \left(\theta_{k}-\theta_{m}\right)\right] \\
P_{m} & =\frac{k_{c}}{\left(k_{c}-1\right)} V_{m} V_{k} B_{\mathrm{km}} \sin \left(\theta_{m}-\theta_{k}\right) \\
Q_{m} & =\frac{k_{c}}{\left(k_{c}-1\right)} B_{\mathrm{km}}\left[V_{m}^{2}-V_{m} V_{k} \cos \left(\theta_{m}-\theta_{k}\right)\right]
\end{aligned}
$$

where the per-unit compensation $\left(k_{c}\right)$ is defined as $k_{c}=$ $\left(X_{C} / X_{L}\right), X_{L}$ is the reactance of the line, and $B_{\mathrm{km}}=\left(1 / X_{L}\right)$.

The dynamic characteristics of a TCSC, as shown in Fig. 3, is assumed to be modeled by a single time constant $\left(T_{\mathrm{tcsc}}=\right.$ 


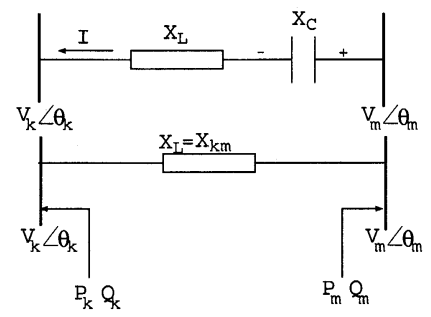

Fig. 2. Power injection model of TCSC.

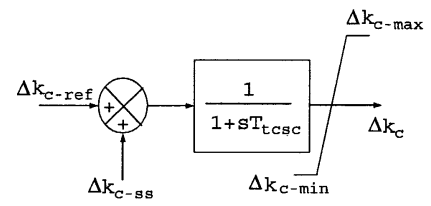

Fig. 3. Small-signal dynamic model of TCSC.

$0.02 \mathrm{~s}$ ) representing the response time of the TCSC control circuit as follows:

$$
\frac{d}{d t} \Delta k_{c}=\frac{1}{T_{\mathrm{tcsc}}}\left(-\Delta k_{c}+\Delta k_{c-\text { ref }}+\Delta k_{c-s s}\right) .
$$

In Fig. $3, \Delta k_{c}$ is the incremental change in value of $k_{c}$ about the nominal value of 0.5 and $\Delta k_{c-\text { ref }}$ is the reference setting which is augmented by $\Delta k_{c-s s}$ in the presence of supplementary damping control. In the small-signal model of the TCSC, we have represented device saturation by incorporating realistic limits $(0.25-0.75)$ on the output. The overall transport delays for the remote signals were modeled by a first-order filter $\left(1 /\left(1+T_{d} s\right)\right)$ in the feedback path where $T_{d}$ is the equivalent time constant representing the delay. The value of $T_{d}$ can vary depending on the distance of the controller site from the remote signal sensor location and the mechanism of data acquisition, conditioning, and transmission. We have chosen the value of $T_{d}$ to be $0.05 \mathrm{~s}$.

A successive relaxation algorithm [13] was employed to determine the steady-state settings of these devices to meet the desired line flows. The nodal voltage magnitudes and angles were solved by the conventional N-R load flow while a separate subproblem was solved at the end of each N-R iteration to update the state variables for the FACTS in order to meet the specified line-flow criteria. The iterative process converged when both the load-flow and the line-flow criteria were satisfied. This avoids ill-conditioning of the power flow Jacobian.

The machine, exciter, network power flow, and power injection model for the FACTS devices were linearized around the nominal operating condition to produce the linear dynamic model for eigenanalysis and control design.

\section{Centralized Damping Control Design: MIXED-SENSITIVITY-BASED LMI APPROACH}

Oscillations in power systems are triggered by sudden variations in load demand, action of voltage regulators due to faults, etc. The primary function of the damping controllers is to minimize the impact of these disturbances on the system. Fig. 4 displays the output disturbance rejection problem in the standard

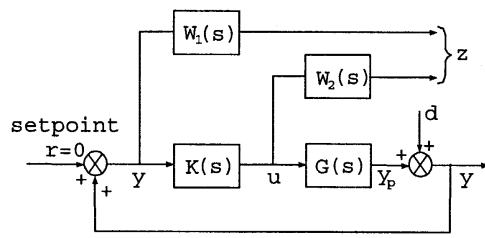

Fig. 4. Mixed-sensitivity output disturbance rejection configuration.

mixed-sensitivity configuration where $G(s)$ is the open-loop plant, $K(s)$ is the controller to be designed, and $W_{1}(s)$ and $W_{2}(s)$ are weights for shaping the characteristics of the open-loop plant. The design objective is to minimize a weighted mix of the transfer function $S(s)=(I-G(s) K(s))^{-1}$, which ensures disturbance rejection and $K(s) S(s)=$ $-G(s) K(s))^{-1}$ which handles the robustness issues and minimizes the control effort. This mixed-sensitivity $(S / K S)$ design objective is represented in [14] as

$$
\left\|\left[\begin{array}{c}
W_{1}(s) S(s) \\
W_{2}(s) K(s) S(s)
\end{array}\right]\right\|_{\infty}<1
$$

The state-space description of the augmented-plant is given by

$$
\left[\begin{array}{c}
\dot{\mathbf{x}}_{\mathbf{p}} \\
\mathbf{z} \\
\mathbf{y}
\end{array}\right]=\left[\begin{array}{ccc}
A & B_{1} & B_{2} \\
C_{1} & D_{11} & D_{12} \\
C_{2} & D_{21} & 0
\end{array}\right]\left[\begin{array}{c}
\mathbf{x}_{\mathbf{p}} \\
\mathbf{d} \\
\mathbf{u}
\end{array}\right]
$$

where $\mathbf{x}_{\mathbf{p}}$ is the state variable vector of the plant $G(s)$ and weights $\left(W_{1}(s), W_{2}(s)\right)$ combined, $\mathbf{d}$ is the disturbance input, $\mathbf{u}$ is the plant input, $\mathbf{y}_{\mathbf{p}}$ is the plant output, $\mathbf{y}$ is the measured signals including disturbances and $\mathbf{z}$ is the regulated output. The state-space representation of the controller is given by

$$
\begin{aligned}
\dot{\mathbf{x}}_{\mathbf{k}} & =A_{k} \mathbf{x}_{\mathbf{k}}+B_{k} \mathbf{y} \\
\mathbf{u} & =C_{k} \mathbf{x}_{\mathbf{k}}+D_{k} \mathbf{y}
\end{aligned}
$$

where $\mathbf{x}_{\mathbf{k}}$ represents the controller states; $\mathbf{u}$ is the controller output that links with $\Delta k_{c-s s}$ in (5); $\Delta P_{51,45}, \Delta P_{18,16}$, and $\Delta P_{13,17}$ are the perturbations of the plant outputs $\left(\mathbf{y}_{\mathbf{p}}\right) P_{51,45}$, $P_{18,16}$, and $P_{13,17}$ around the nominal operating point, respectively. The transfer matrix between $\mathbf{d}$ and $\mathbf{z}$ is given by

$$
T_{z d}(s)=\left[\begin{array}{c}
W_{1}(s) S(s) \\
W_{2}(s) K(s) S(s)
\end{array}\right]=C_{c l}\left(s I-A_{c l}\right)^{-1} B_{c l}+D_{c l}
$$

where

$$
\begin{aligned}
& A_{c l}=\left[\begin{array}{cc}
A+B_{2} D_{k} C_{2} & B_{2} C_{k} \\
B_{k} C_{2} & A_{k}
\end{array}\right] \\
& B_{c l}=\left[\begin{array}{c}
B_{1}+B_{2} D_{k} D_{21} \\
B_{k} D_{21}
\end{array}\right] \\
& C_{c l}=\left[C_{1}+D_{12} D_{k} C_{2} \quad D_{12} C_{k}\right] \\
& D_{c l}=D_{11}+D_{12} D_{k} D_{21} \text {. }
\end{aligned}
$$

The bounded real lemma, discussed in [15], with the help of Schur's formula for the determinant of a partitioned matrix [14], 
allows us to conclude that the closed-loop system in (10) is asymptotically stable if there exists an $X=X^{T}>0$ such that

$$
\left[\begin{array}{ccc}
A_{c l}^{T} X+X A_{c l} & B_{c l} & X C_{c l}^{T} \\
B_{c l}^{T} & -I & D_{c l}^{T} \\
C_{c l} X & D_{c l} & -I
\end{array}\right]<0 .
$$

In other words, $\left\|T_{z d}\right\|_{\infty}<1$, with guaranteed asymptotic stability, is equivalent to the existence of $X=X^{T}>0$ that satisfies the LMI condition in (15). Hence, the controller design problem boils down to solving this LMI. However, inequality (15) contains $A_{c l} X$ and $C_{c l} X . A_{c l}$ and $C_{c l}$ are functions of the controller parameters $A_{k}, B_{k}, C_{k}$ and $D_{k}$ and the controller parameters themselves are functions of $X$ making the products $A_{c l} X, C_{c l} X$ nonlinear in $X$. To convert the problem into a linear one, a change of controller variables is necessary. The new controller variables are given by (16)-(19) where $R, S, M$, and $N$ are submatrices of $X$ [16], [17]

$$
\begin{aligned}
\hat{A}= & N A_{k} M^{T}+N B_{k} C_{2} R+S B_{2} C_{k} M^{T} \\
& +S\left(A+B_{2} D_{k} C_{2}\right) R \\
\hat{B}= & N B_{k}+S B_{2} D_{k} \\
\hat{C}= & C_{k} M^{T}+D_{k} C_{2} R \\
\hat{D}= & D_{k} .
\end{aligned}
$$

The necessary transformation through substitution of new controller variables requires a solution to the design problem given by

$$
\begin{aligned}
& {\left[\begin{array}{cc}
R & I \\
I & S
\end{array}\right]>0} \\
& {\left[\begin{array}{ll}
\Psi_{11} & \Psi_{21}^{T} \\
\Psi_{21} & \Psi_{22}
\end{array}\right]<0}
\end{aligned}
$$

where

$$
\begin{aligned}
\Psi_{11} & =\left[\begin{array}{cc}
A R+R A^{T}+B_{2} \hat{C}+\hat{C}^{T} B_{2}^{T} & B_{1}+B_{2} \hat{D} D_{21} \\
\left(B_{1}+B_{2} \hat{D} D_{21}\right)^{T} & I
\end{array}\right] \\
\Psi_{21} & =\left[\begin{array}{cc}
\hat{A}+\left(A+B_{2} \hat{D} C_{2}\right)^{T} & S B_{1}+\hat{B} D_{21} \\
C_{1} R+D_{12} \hat{C} & D_{11}+D_{12} \hat{D} D_{21}
\end{array}\right] \\
\Psi_{22} & =\left[\begin{array}{cc}
A^{T} S+S A+\hat{B} C_{2}+C_{2}^{T} \hat{B}^{T} & \left(C_{1}+D_{12} \hat{D} C_{2}\right)^{T} \\
C_{1}+D_{12} \hat{D} C_{2} & -I
\end{array}\right] .
\end{aligned}
$$

The LMIs in (20)-(21) are solved for $\hat{A}, \hat{B}, \hat{C}$, and $\hat{D}$ as an optimization problem. Once $\hat{A}, \hat{B}, \hat{C}$, and $\hat{D}$ are obtained, $A_{k}$, $B_{k}, C_{k}$, and $D_{k}$ are recovered from $\hat{A}, \hat{B}, \hat{C}$, and $\hat{D}$ by solving (16)-(19).

\section{Centralized Design of Damping Controller FOR TCSC}

The LMI formulation in Section IV produces centralized controllers in multivariable form. Here a three-input, one-output controller was designed for the TCSC using three stabilizing signals from three different remote locations. The system has 133 states. To expedite the process in the LMI routine, the plant order was reduced to 10. The Robust Control Toolbox available with Matlab [18] was used to perform the necessary computations. Balanced truncation [14], [19] was used for the reduction of the plant model. An order of 10 was found to be satisfactory in both prefault and postfault operating conditions.

In a Riccati-based approach, the standard practice is to choose the weight $W_{1}(s)$ as a high-gain low-pass filter for output disturbance rejection. The weight $W_{2}(s)$ should be a high-pass filter in order to reduce the control effort and to ensure robustness against additive uncertainties in the plant model in the high-frequency range. As a starting point, we have chosen the same shape of weights $W_{1}(s)$ and $W_{2}(s)$ used in [9] for superconducting magnetic energy storage (SMES) control design. A scale factor of 0.8475 was found to suit the design requirement for the TCSC. The weights $W_{1}(s)$ and $W_{2}(s)$ are given by

$$
\begin{aligned}
& W_{1}(s)=0.8475 \frac{99 s+11400}{s^{2}+156 s+12504} \\
& W_{2}(s)=0.8475 \frac{0.1055 s^{2}+0.037 s+0.0094}{s^{2}+0.0020 s} .
\end{aligned}
$$

The multiobjective (disturbance rejection and control effort optimization) feature of LMI was accessed through suitably defined objective in the argument of the function hinfmix of the LMI Toolbox in Matlab [18]. The order of the controller obtained from the LMI solution was equal to the reduced plant order plus the order of the weights, which was quite high from a practical implementation point of view. Therefore, the controller was reduced to a sixth-order one by the balanced truncation without significantly affecting the frequency response. This reduced-order controller was tested on the original system (full order) model for both prefault and postfault operating conditions. Linear simulation was carried out using the function lsim in Matlab to examine the settling of the oscillations. A 10-s settling time was set as the criterion which was achieved through slight adjustment in the gain of the controllers. A 10-s settling time in linear simulation generally guarantees, in the experience of the authors, that oscillations will settle down in 10-15 s in nonlinear simulations, which is adopted by many utilities in their system design and operation guidelines [1]. The state variable representation of the three-input, one-output controller for the TCSC is given in the Appendix A.

\section{ROBUSTNESS VALIDATION AND PERFORMANCE EVALUATION}

An eigenanalysis of the system was carried out to examine the performance of the controller under different operating scenarios. The results are shown in Tables Ito IV for different operating conditions. It is clear from these results that the damping ratios of the interarea modes in the presence of the controller are improved considerably. Although the damping of the fourth mode looks low, it is adequate as the time-domain simulation shows that oscillations influenced by this mode settle in 10-12 s. Table I contains the results in open-loop and closed-loop under both prefault and postfault conditions. It can be seen that the damping of modes \#1, \#2, and \#3, shown in boldface, is improved considerably in the presence of the controller. The damping action of the controller was examined at different power-flow levels. Table II displays the damping ratios and frequencies of the interarea modes when power flow from NETS to NYPS varies in the range 
TABLE II

DAMPING RATIOS AND FREQUENCIES OF INTERAREA MODES AT DIFFERENT LEVELS OF POWER-FLOW BETWEEN NETS AND NYPS

\begin{tabular}{c|c|c|c|c|c|c|c|c}
\hline \multirow{2}{*}{$\begin{array}{c}\text { Power flow } \\
(\mathrm{MW})\end{array}$} & \multicolumn{2}{|c|}{ Mode 1 } & \multicolumn{2}{c|}{ Mode 2} & \multicolumn{2}{c|}{ Mode 3 } & \multicolumn{2}{c}{ Mode4 } \\
\cline { 2 - 8 } & $\zeta$ & $\mathrm{f}(\mathrm{Hz})$ & $\zeta$ & $\mathrm{f}(\mathrm{Hz})$ & $\zeta$ & $\mathrm{f}(\mathrm{Hz})$ & $\zeta$ & $\mathrm{f}(\mathrm{Hz})$ \\
\hline 100 & $\mathbf{0 . 3 8 8 9}$ & 0.3360 & $\mathbf{0 . 1 2 4 1}$ & 0.4756 & $\mathbf{0 . 1 0 3 9}$ & 0.6393 & 0.0518 & 0.7912 \\
\hline 500 & $\mathbf{0 . 3 6 5 9}$ & 0.3383 & $\mathbf{0 . 1 2 9 0}$ & 0.4815 & $\mathbf{0 . 1 0 8 4}$ & 0.6230 & 0.0518 & 0.7910 \\
\hline 700 & $\mathbf{0 . 3 5 4 5}$ & 0.3401 & $\mathbf{0 . 1 3 3 0}$ & 0.4853 & $\mathbf{0 . 1 1 2 0}$ & 0.6116 & 0.0518 & 0.7909 \\
\hline 900 & $\mathbf{0 . 3 4 4 1}$ & 0.3426 & $\mathbf{0 . 1 3 9 0}$ & 0.4909 & $\mathbf{0 . 1 1 6 8}$ & 0.5967 & 0.0518 & 0.7907 \\
\hline
\end{tabular}

TABLE III

DAMPING RATIOS AND FREQUENCIES OF INTERAREA MODES FOR DIFFERENT LOAD CHARACTERISTICS

\begin{tabular}{c|c|c|c|c|c|c|c|c}
\hline \multirow{2}{*}{$\begin{array}{c}\text { Type of } \\
\text { load }\end{array}$} & \multicolumn{2}{|c|}{ Mode 1 } & \multicolumn{2}{c|}{ Mode 2 } & \multicolumn{2}{c|}{ Mode 3 } & \multicolumn{2}{c}{ Mode4 } \\
\cline { 2 - 8 } CI & $\zeta$ & $\mathrm{f}(\mathrm{Hz})$ & $\zeta$ & $\mathrm{f}(\mathrm{Hz})$ & $\zeta$ & $\mathrm{f}(\mathrm{Hz})$ & $\zeta$ & $\mathrm{f}(\mathrm{Hz})$ \\
\hline $\mathrm{CC}$ & $\mathbf{0 . 3 5 4 5}$ & 0.3401 & $\mathbf{0 . 1 3 3 0}$ & 0.4853 & $\mathbf{0 . 1 1 2 0}$ & 0.6116 & 0.0518 & 0.7909 \\
\hline $\mathrm{CC}+\mathrm{CI}$ & $\mathbf{0 . 3 7 5 9}$ & 0.3537 & $\mathbf{0 . 1 4 9 8}$ & 0.4846 & $\mathbf{0 . 1 0 7 1}$ & 0.6134 & 0.0520 & 0.7914 \\
\hline $\mathrm{CP}+\mathrm{CI}$ & $\mathbf{0 . 3 7 2 8}$ & 0.3514 & $\mathbf{0 . 1 4 5 6}$ & 0.4849 & $\mathbf{0 . 1 0 8 3}$ & 0.6129 & 0.0520 & 0.7913 \\
\hline Dynamic & $\mathbf{0 . 3 5 9 4}$ & 0.3444 & $\mathbf{0 . 1 3 2 9}$ & 0.4853 & $\mathbf{0 . 1 2 5 1}$ & 0.6107 & 0.0519 & 0.7908 \\
\hline
\end{tabular}

TABLE IV

DAMPING RATIOS AND FREQUENCIES OF INTERAREA MODES FOR DiFFERENT TIE-LINE STRENGTHS

\begin{tabular}{c|c|c|c|c|c|c|c|c}
\hline \multirow{2}{*}{$\begin{array}{c}\text { Outage of } \\
\text { tie-line }\end{array}$} & \multicolumn{2}{|c|}{ Mode 1 } & \multicolumn{2}{c|}{ Mode 2 } & \multicolumn{2}{c|}{ Mode 3 } & \multicolumn{2}{c}{ Mode4 } \\
\cline { 2 - 9 } & $\zeta$ & $\mathrm{f}(\mathrm{Hz})$ & $\zeta$ & $\mathrm{f}(\mathrm{Hz})$ & $\zeta$ & $\mathrm{f}(\mathrm{Hz})$ & $\zeta$ & $\mathrm{f}(\mathrm{Hz})$ \\
\hline $60-61$ & $\mathbf{0 . 2 7 9 3}$ & 0.3529 & $\mathbf{0 . 1 3 4 8}$ & 0.5308 & $\mathbf{0 . 2 0 0 5}$ & 0.5375 & 0.0519 & 0.7907 \\
\hline $53-54$ & $\mathbf{0 . 2 7 1 6}$ & 0.3433 & $\mathbf{0 . 1 2 5 8}$ & 0.5313 & $\mathbf{0 . 1 5 6 4}$ & 0.5485 & 0.0520 & 0.7905 \\
\hline $27-53$ & $\mathbf{0 . 3 3 5 0}$ & 0.3386 & $\mathbf{0 . 1 4 0 6}$ & 0.4925 & $\mathbf{0 . 1 2 0 3}$ & 0.5963 & 0.0519 & 0.7907 \\
\hline
\end{tabular}

100-900 MW. The damping is found to be highly satisfactory in all cases. The performance of the controller was further evaluated with various load models using constant impedance (CI), a mixture of constant current and constant impedance $(\mathrm{CC}+\mathrm{CI})$, a mixture of constant power and constant impedance $(\mathrm{CP}+\mathrm{CI})$, and dynamic load characteristics. The dynamic load (induction motor type) was considered to be at bus \#17, the remainder being of CI type. It is evident from the results of Table III that the designed controller provides robust damping for these different load characteristics. Table IV demonstrates the robustness of the damping action against outage of different tie-lines connecting NETS and NYPS. The damping action is found to be quite robust with respect to the outage of each of the tie-lines between buses \#27-\#53, buses \#60-\#61, and buses \#53-\#54 connecting NETS and NYPS. The damping performance of the designed controller was therefore found to be robust against widely varying operating conditions. One of the concerns of centralized design using remote signals is the possible loss of one of the channels leading to unsatisfactory damping performance. Reference [20] has proposed a solution based on the replacement of the lost remote signal by a similar local signal through the use of a signal-loss detector. It is possible to adjust the phase characteristics of the replacement local signal by a prefilter of required gain and phase. However, we have not investigated further into the matter in this research.

A nonlinear simulation was carried out for $30 \mathrm{~s}$ to further demonstrate performance robustness of the controllers in the presence of system nonlinearities, including saturation. Simultaneous integration technique for solving differential and algebraic equations using the trapezoidal rule was used and coded for running the simulations in Matlab. One of the most probable contingencies of the system that triggers interarea oscillations is a three-phase solid fault near bus \#53 on one of the tie-lines
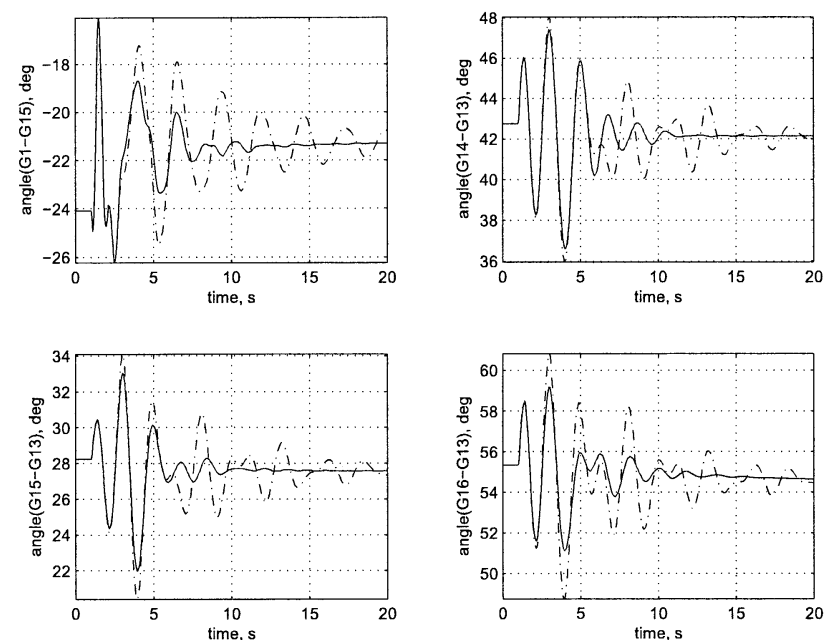

Fig. 5. Dynamic response of the system with centralized control using global signals, solid line: with controller; dashed line: without controller.

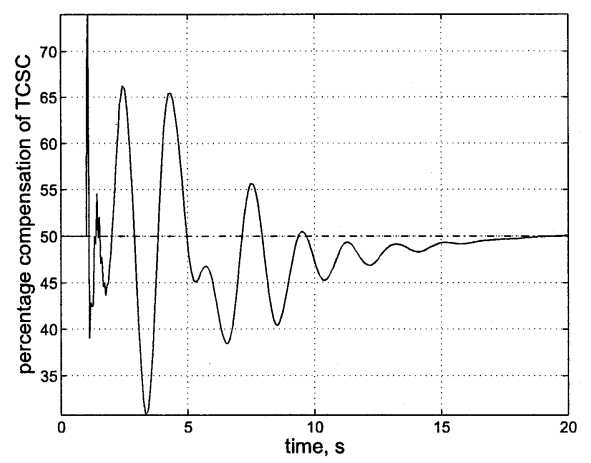

Fig. 6. Dynamic response of the TCSC with centralized control, solid line: with controller: dashed line; without controller.

connecting buses \#53-\#54. The fault condition was simulated after $1 \mathrm{~s}$ for a duration of $80 \mathrm{~ms}(\approx 5$ cycles in a $60-\mathrm{Hz}$ system $)$ followed by opening of the faulted line. The dynamic response of the system model in terms of the relative angular separation of G1 with respect to machine G15 and that of G14, G15, and G16 with respect to G13 following this contingency is shown in Fig. 5. In these figures, "without controller" means the absence of supplementary damping control although the primary control loop for the FACTS device continues to maintain steady-state power-flow requirements. Fig. 5 illustrates that the interarea oscillations are damped out in 10-15 s through control action. Fig. 6 shows the output response of the TCSC. It is to be noted that the response of the damping controller for the TCSC is similar to the output of the TCSC without the steady-state offset. It is observed that the output of the TCSC is within $25 \%$ to $75 \%$, a compensation range which can easily be achieved by TCSC manufacturers.

\section{Decentralized Design With Local Signal}

We have also carried out the damping control design for the same study system employing only local stabilizing signals. In this case, the supplementary damping control action was required through two additional FACTS devices in order to damp 
TABLE V

DAMPING RATIOS AND FREQUENCIES OF INTERAREA MODES WITH THE DECENTRALIZED CONTROLLER FOR TCSC USING LOCAL SIGNAL

\begin{tabular}{c|c|c|c|c|c|c|c|c}
\hline \multirow{2}{*}{ Mode No. } & \multicolumn{4}{|c|}{ Pre-fault } & \multicolumn{4}{c}{ Post-fault } \\
\cline { 2 - 9 } & \multicolumn{2}{|c|}{ Open-loop } & \multicolumn{2}{c|}{ Closed-loop } & \multicolumn{3}{c}{ Open-loop } & \multicolumn{2}{c}{ Closed-loop } \\
\cline { 2 - 9 } & $\zeta$ & $\mathrm{f}(\mathrm{Hz})$ & $\zeta$ & $\mathrm{f}(\mathrm{Hz})$ & $\zeta$ & $\mathrm{f}(\mathrm{Hz})$ & $\zeta$ & $\mathrm{f}(\mathrm{Hz})$ \\
\hline 1 & $\mathbf{0 . 0 6 2 6}$ & 0.3945 & $\mathbf{0 . 1 5 4 4}$ & 0.3434 & $\mathbf{0 . 0 5 1 3}$ & 0.3759 & $\mathbf{0 . 1 3 1 0}$ & 0.3288 \\
\hline 2 & 0.0434 & 0.5105 & 0.0545 & 0.4991 & 0.0422 & 0.5094 & 0.0543 & 0.5002 \\
\hline 3 & 0.0560 & 0.6269 & 0.0656 & 0.6191 & 0.0476 & 0.5978 & 0.0618 & 0.5841 \\
\hline 4 & 0.0499 & 0.7923 & 0.0502 & 0.7918 & 0.0499 & 0.7920 & 0.0502 & 0.7914 \\
\hline
\end{tabular}

TABLE VI

DAMPING RATIOS AND FREQUENCIES OF INTERAREA MODES WiTH THE DECENTRALIZED CONTROLLERS FOR TCSC AND SVC USING LOCAL SIGNAL

\begin{tabular}{c|c|c|c|c|c|c|c|c}
\hline \multirow{2}{*}{ Mode No. } & \multicolumn{4}{|c|}{ Pre-fault } & \multicolumn{4}{c}{ Post-fault } \\
\cline { 2 - 9 } & \multicolumn{2}{|c|}{ Open-loop } & \multicolumn{2}{c|}{ Closed-loop } & \multicolumn{2}{c}{ Open-loop } & \multicolumn{2}{c}{ Closed-loop } \\
\cline { 2 - 9 } & $\zeta$ & $\mathrm{f}(\mathrm{Hz})$ & $\zeta$ & $\mathrm{f}(\mathrm{Hz})$ & $\zeta$ & $\mathrm{f}(\mathrm{Hz})$ & $\zeta$ & $\mathrm{f}(\mathrm{Hz})$ \\
\hline 1 & 0.1544 & 0.3434 & 0.1795 & 0.3158 & 0.1310 & 0.3288 & 0.1464 & 0.3060 \\
\hline 2 & $\mathbf{0 . 0 5 4 5}$ & 0.4991 & $\mathbf{0 . 1 0 3 1}$ & 0.4549 & $\mathbf{0 . 0 5 4 3}$ & 0.5002 & $\mathbf{0 . 1 0 9 1}$ & 0.4536 \\
\hline 3 & 0.0656 & 0.6191 & 0.0643 & 0.6184 & 0.0618 & 0.5841 & 0.0584 & 0.5835 \\
\hline 4 & 0.0502 & 0.7918 & 0.0603 & 0.7864 & 0.0502 & 0.7914 & 0.0603 & 0.7860 \\
\hline
\end{tabular}

TABLE VII

DAMPING RATIOS AND FREQUENCIES OF INTERAREA MODES With THE DECENTRALIZED CONTROLLERS FOR TCSC, SVC, AND CPS USING LOCAL SIGNAL

\begin{tabular}{|c|c|c|c|c|c|c|c|c|}
\hline \multirow{2}{*}{ Mode No. } & \multicolumn{4}{|c|}{ Pre-fault } & \multicolumn{4}{c|}{ Post-fault } \\
\cline { 2 - 9 } & \multicolumn{2}{|c|}{ Open-loop } & \multicolumn{2}{c|}{ Closed-loop } & \multicolumn{2}{c|}{ Open-loop } & \multicolumn{2}{c|}{ Closed-loop } \\
\cline { 2 - 9 } & $\zeta$ & $\mathrm{f}(\mathrm{Hz})$ & $\zeta$ & $\mathrm{f}(\mathrm{Hz})$ & $\zeta$ & $\mathrm{f}(\mathrm{Hz})$ & $\zeta$ & $\mathrm{f}(\mathrm{Hz})$ \\
\hline 1 & 0.1795 & 0.3158 & 0.3140 & 0.2682 & 0.1464 & 0.3060 & 0.2784 & 0.2709 \\
\hline 2 & 0.1031 & 0.4549 & 0.2266 & 0.4444 & 0.1091 & 0.4536 & 0.2060 & 0.4091 \\
\hline 3 & $\mathbf{0 . 0 6 4 3}$ & 0.6184 & $\mathbf{0 . 1 1 0 5}$ & 0.4585 & $\mathbf{0 . 0 5 8 4}$ & 0.5835 & $\mathbf{0 . 1 0 7 6}$ & 0.4539 \\
\hline 4 & 0.0603 & 0.7864 & 0.0600 & 0.7858 & 0.0603 & 0.7860 & 0.0601 & 0.7848 \\
\hline
\end{tabular}

out the three dominant interarea modes. The controllers for the three FACTS devices were designed sequentially (i.e., once the damping controller for one device was designed, the loop was closed for designing the next one). The transfer function representation of the single-input, single-output (SISO) controllers designed for the TCSC, SVC, and CPS are given in the Appendix $\mathrm{B}$. Table $\mathrm{V}$ shows the damping ratios and frequency of oscillations of the interarea modes with the controller for the TCSC only. It can be seen that using the local signal, only the damping of mode \#1 is improved with no significant improvement of modes \#2, \#3, and \#4. An additional controller for the SVC improves the damping of mode \#2 as shown in Table VI. Similarly from Table VII, it is clear that the controller for CPS improves the damping of mode \#3. Thus, with a local stabilizing signal, one separate FACTS device is required to damp out each dominant interarea mode. However, with global signals, one single controller designed for the TCSC produces the desired damping to all poorly damped modes. The results of the nonlinear simulation with the three decentralized controllers for the TCSC, SVC, and CPS are shown in Fig. 7 for the same disturbance considered in the centralized case. The performance is reasonably similar to that of the centralized design employing global signals. From the dynamic response of the system, it is evident that the use of global signals requires only one centralized controller for the TCSC to damp out all three critical interarea modes; whereas, two additional FACTS devices are necessary if local signals are used. This is due to diverse modal content in global signals from several locations as mentioned in [4]. The outputs of the three FACTS devices following the disturbance are shown in Fig. 8.
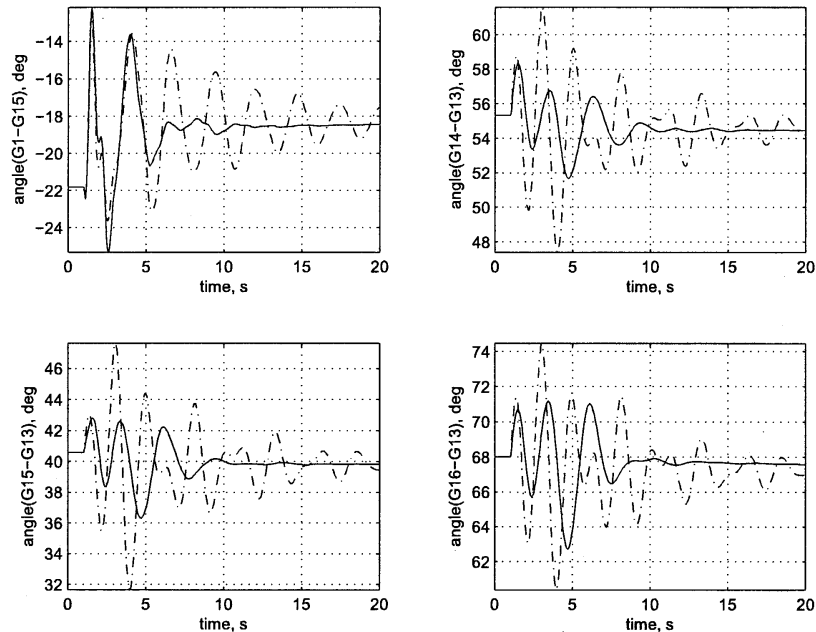

Fig. 7. Dynamic response of the system with decentralized control using local signals, solid line: with controller; dashed line: without controller.
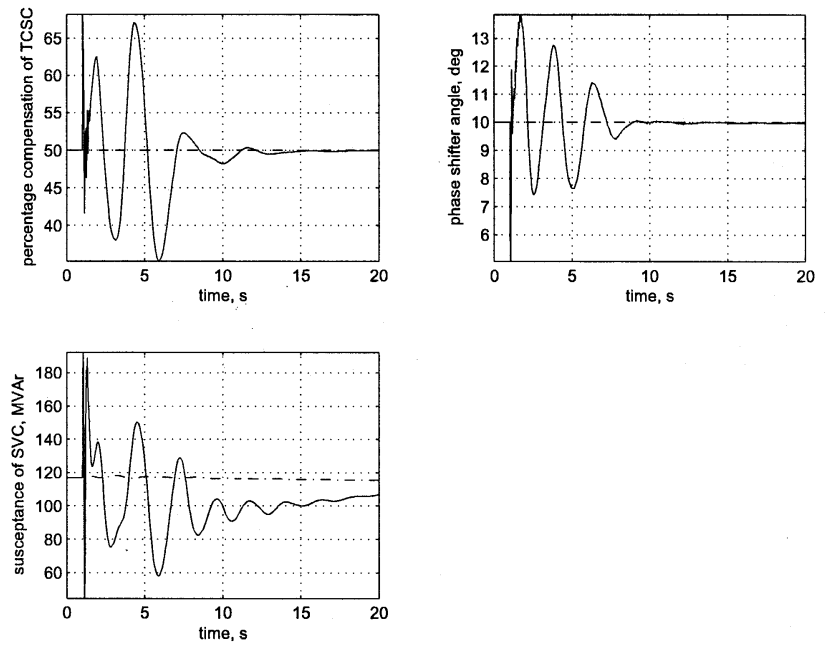

Fig. 8. Dynamic response of the FACTS devices with decentralized control, solid line: with controller; dashed line: without controller.

\section{CONCLUSION}

We have demonstrated the use of global stabilizing signals for effective damping of multiple swing modes through a single TCSC. The centralized design of the multivariable controller has been formulated as a multiobjective optimization problem in the LMI framework. The solution is numerically sought through the LMI solver. The performance robustness of the designed controller has been verified in the frequency domain through eigenanalysis and also in the time domain through nonlinear simulations. The decentralized design, using local signals, is always a better and reliable option. But in practical power systems, the number of dominant interarea modes is often much larger than the number of control devices available and, therefore, centralized control design, using global signals, is one of the potential options worth exploring. With rapid advancements in the field of PMU based wide-area measurement using GPS, real-time delivery of synchronous phasors and control signals has become fairly simple and this has made the prospect of damping of in- 


$$
\begin{aligned}
A_{T C S C} & =1 \times 10^{4}\left[\begin{array}{cccccc}
-6.07 & -0.6048 & -0.0138 & 0.0418 & -0.4443 & -0.0818 \\
0.5617 & -0.0013 & -0.0001 & 0.0004 & -0.0050 & -0.0011 \\
0.0118 & -0.0001 & -0.00001 & 0.0001 & -0.0006 & -0.0001 \\
-0.0219 & 0.0004 & -0.00001 & -0.0002 & 0.0032 & 0.0007 \\
0.3911 & -0.0042 & -0.0005 & 0.0008 & -0.0309 & -0.0091 \\
0.0768 & -0.0008 & -0.0001 & 0.0003 & -0.0086 & -0.0032
\end{array}\right] . \\
B_{T C S C} & =\left[\begin{array}{cccccc}
209.80 & -2.5605 & -0.1591 & 0.5576 & -7.1145 & -1.3732 \\
-175.98 & 2.2237 & 0.1788 & -0.2978 & 6.2594 & 1.3601 \\
-146.52 & -2.0953 & -0.0658 & 0.6446 & -0.9342 & 0.2312
\end{array}\right]^{T} \\
C_{T C S C} & =\left[\begin{array}{llllll}
-310.58 & -3.9864 & -0.2482 & 0.9028 & -9.5220 & -1.9465
\end{array}\right]
\end{aligned}
$$

terarea oscillations through global signals a realistic one. The main contribution of this paper is in applying the mixed-sensitivity based LMI design methodology to decentralized and centralized design of FACTS damping controllers and illustrating the use of global stabilizing signals to damp out multiple swing modes employing a single control device.

\section{APPENDIX A \\ MISO CONTROLLER}

The state-space representation of the three-input, one-output centralized controller for the TCSC is given top. (See the equation shown top.)

\section{APPENDIX B \\ SISO CONTROLLER}

The transfer function representations of the single-input, single-output controllers for the TCSC, SVC, and CPS are given below

$$
\begin{aligned}
K_{\mathrm{TCSC}}(s)= & K_{\mathrm{TCSC}} \frac{N_{\mathrm{TCSC}}(s)}{D_{\mathrm{CSC}}(s)}, \quad K_{\mathrm{TCSC}}=1.05 \\
N_{\mathrm{TCSC}}(s)= & -4.88 \times 10^{5} s^{4}-2.33 \times 10^{8} s^{3} \\
& -2.76 \times 10^{9} s^{2}-1.55 \times 10^{10} s+1.56 \times 10^{9} \\
D_{\mathrm{TCSC}}(s)= & s^{5}+2.14 \times 10^{5} s^{4}+1.77 \times 10^{8} s^{3} \\
& +1.32 \times 10^{10} s^{2}+6.51 \times 10^{9} s+2.38 \times 10^{10} \\
K_{\mathrm{SVC}}(s)= & K_{\mathrm{SVC}} \frac{N_{\mathrm{SVC}}(s)}{D_{\mathrm{SVC}}(s)}, \quad K_{\mathrm{SVC}}=2.70 \\
N_{\mathrm{SVC}}(s)= & 8.36 \times 10^{3} s^{4}+7.51 \times 10^{6} s^{3} \\
& -3.31 \times 10^{7} s^{2}+1.27 \times 10^{9} s-3.43 \times 10^{8} \\
D_{\mathrm{SVC}}(s)= & s^{5}+7.79 \times 10^{3} s^{4}+9.47 \times 10^{6} s^{3} \\
& +5.35 \times 10^{8} s^{2}+1.71 \times 10^{9} s+2.09 \times 10^{9} \\
K_{\mathrm{CPS}}(s)= & K_{\mathrm{CPS}} \frac{N_{\mathrm{CPS}}(s)}{D_{\mathrm{CPS}}(s)}, \quad K_{\mathrm{CPS}}=1.00 \\
N_{\mathrm{CPS}}(s)= & 1.02 \times 10^{6} s^{4}+1.83 \times 10^{9} s^{3} \\
& +1.01 \times 10^{11} s^{2}+3.05 \times 10^{11} s+5.36 \times 10^{10} \\
D_{\mathrm{CPS}}(s)= & s^{5}+8.94 \times 10^{5} s^{4}+8.87 \times 10^{9} s^{3} \\
& +3.69 \times 10^{12} s^{2}+2.56 \times 10^{12} s+2.26 \times 10^{12} .
\end{aligned}
$$

\section{ACKNOWLEDGMENT}

The authors gratefully acknowledge the help and suggestions received from Prof. B. J. Cory, Dr. I. M. Jaimoukha, Dr. T. C. Green, and Dr. A. C. Zolotas during this research.

\section{REFERENCES}

[1] J. Paserba, Analysis and control of power system oscillation, in CIGRE Special Publication 38.01.07, vol. Tech. Brochure 111, 1996.

[2] P. Kundur, Power System Stability and Control. New York: McGrawHill, 1994.

[3] N. Hingorani and L. Gyugyi, Understanding FACTS. New York: IEEE Press, 2000

[4] J. Chow, J. Sanchez-Gasca, H. Ren, and S. Wang, "Power system damping controller design using multiple input signals," IEEE Contr. Syst. Mag., vol. 20, pp. 82-90, Aug. 2000.

[5] I. Kamwa, R. Grondin, and Y. Hebert, "Wide-area measurement based stabilizing control of large power systems-A decentralized/hierarchical approach," IEEE Trans. Power Syst., vol. 16, pp. 136-153, Feb. 2001.

[6] G. Heydt, C. Liu, A. Phadke, and V. Vittal, "Solutions for the crisis in electric power supply," IEEE Comput. Appl. Power, vol. 14, pp. 22-30, July 2001

[7] Z. Xie, G. Manimaran, V. Vittal, A. G. Phadke, and V. Centeno, "An information architecture for future power systems and its reliability analysis," IEEE Trans. Power Syst., vol. 17, pp. 857-863, Aug. 2002.

[8] M. Aboul-Ela, A. Sallam, J. McCalley, and A. Fouad, "Damping controller design for power system oscillations using global signals," IEEE Trans. Power Syst., vol. 11, pp. 767-773, May 1996.

[9] B. Pal, A. Coonick, and B. Cory, "Robust damping of interarea oscillations in power systems with superconducting magnetic energy storage devices," Proc. Inst. Elect. Eng., Gen. Transm. Dist., vol. 146, no. 06, pp. 633-639, Nov. 1999.

[10] G. Rogers, Power System Oscillations. Norwell, MA: Kluwer, 2000.

[11] N. Martins and L. Lima, "Determination of suitable locations for power system stabilizers and static var compensators for damping electromechanical oscillations in large power systems," IEEE Trans. Power Syst., vol. 5, pp. 1455-1469, Nov. 1990.

[12] M. Noroozian, L. Angquist, M. Gandhari, and G. Andersson, "Improving power system dynamics by series-connected FACTS devices," IEEE Trans. Power Delivery, vol. 12, pp. 1635-1641, Oct. 1997.

[13] M. Noroozian and G. Andersson, "Power flow control by use of controllable series components," IEEE Trans. Power Delivery, vol. 8, pp. 1420-1429, July 1993.

[14] S. Skogestad and I. Postlethwaite, Multivariable Feedback Control. New York: Wiley, 2001.

[15] P. Gahinet and P. Apkarian, "A linear matrix inequality approach to $H_{\infty}$ control," Int. J. Robust Nonlinear Contr., vol. 4, no. 4, pp. 421-448, Apr. 1994.

[16] M. Chilali and P. Gahinet, "Multi-objective output feedback control via LMI optimization," IEEE Trans. Automat. Contr., vol. 42, pp. 896-911, July 1997.

[17] C. Scherer, P. Gahinet, and M. Chilali, " $H_{\infty}$ design with pole placement constraints: an LMI approach," IEEE Trans. Automat. Contr., vol. 41, pp. 358-367, Mar. 1996.

[18] Matlab Users Guide, The Math Works Inc., USA, 1998. 
[19] K. Zhou, J. Doyle, and K. Glover, Robust and Optimal Control. Englewood Cliffs, NJ: Prentice-Hall, 1995.

[20] K. Mekki, A. Snyder, N. HadjSaid, R. Feuillet, D. Georges, and T. Margotin, "Damping controller input-signal loss effects on the wide-area stability of an interconnected power systems," in IEEE Power Eng. Soc. Summer Meeting, vol. 2, 2000, pp. 1015-1019.

Balarko Chaudhuri (S'02) received the B.E.E. (Hons.) degree from Jadavpur University, Calcutta, India, in 2000, and the M.Tech degree from the Indian Institute of Technology, Kanpur, India, in 2002. He is currently pursuing the $\mathrm{Ph}$.D. degree in the control and power field at Imperial College, London, U.K.
Bikash C. Pal (SM'02) received the B.E.E. (Hons.) degree from Jadavpur University, Calcutta, India, in 1990, and the M.E. degree from the Indian Institute of Science, Bangalore, India, in 1992. He received the Ph.D. degree from Imperial College, London, U.K., in 1999.

Currently, he is a Lecturer in the Department of Electrical and Electronic Engineering at Imperial College. His research interests include power system dynamics and FACTS controllers. 